\title{
Determinantes de la Satisfacción Sexual: Personalidad Sexual, Comunicación Emocional y Estrategias de Seducción
}

\author{
Determinants of Sexual Satisfaction: Sexual Personality, \\ Emotional Communication and Seduction Strategies
}

Daniela Judith Cruz Vargas¹, Rozzana Sánchez Aragón²

\begin{abstract}
RESUMEN
La vida sexual de la pareja es central en el entendimiento de su dinámica. Variables percibidas en la otra persona, como rasgos de personalidad sexual, conductas comunicativas y las estrategias con las que seduce a su pareja, son fundamentales en la evaluación subjetiva de la forma, frecuencia y técnicas utilizadas durante la interacción sexual. Ante esto, el presente estudio tuvo como objetivo determinar la relación entre dichas variables en una muestra no probabilística propositiva de 470 hombres y mujeres de la Ciudad de México que reportaron haber sentido pasión por alguien. Algunos de los resultados muestran que, para ambos sexos, los ingredientes esenciales en la experimentación de gozo sexual son: la percepción de personalidades involucradas emocionalmente, atractivas sexuales y dispuestas eróticamente, la ejecución de estrategias de expresión sexual, búsqueda de cercanía, expresión y desenvolvimiento y enamoramiento, la percepción de promiscuidad o restricción. Sin embargo, el uso de las mismas estrategias de seducción favorece la evasión, presión y aburrimiento.
\end{abstract}

Palabras clave: Satisfacción sexual, Personalidad, Emociones, Seducción.

\begin{abstract}
The sexual life of the couple is central in understanding their dynamics. Variables perceived in the other as: sexual personality traits, communicative behaviors and the strategies with which she seduces her partner, are fundamental in the subjective evaluation of the form, frequency and techniques used during sexual interaction. Given this, the present study aimed to determine the relationship between these variables in a non-probabilistic sample of 470 men and women from Mexico City who reported having felt passion for someone. Results show that for both sexes, the essential ingredients in the experimentation of sexual pleasure are the perception of emotionally involved, sexually attractive and erotically disposed personalities, the execution of strategies of sexual expression, search for closeness, expression and development and infatuation, the perception of promiscuity or restriction and the use of the same seduction strategies favors evasion, pressure and boredom.
\end{abstract}

Keywords: Sexual satisfaction, Personality, Emotions, Seduction.
Recibido: 21/05/2018

Aceptado: 13/11/2018
Citar: Cruz Vargas, D. J. \& Sánchez Aragón, R. (2018). Determinantes de la satisfacción sexual: personalidad sexual, comunicación emocional y estrategias de seducción. Investigaciones en Psicología, 23(2), pp. 63-71. doi:10.32824/investigpsicol.a23n2a6 
Las relaciones de pareja se definen como la serie de interacciones que ocurren a lo largo del tiempo, representan un tipo muy específico y primordial de vinculación siendo quizá la más significativa en la vida de las personas, ya que en ellas se vierten las máximas expectativas y anhelos (Ruiz Becerril, 2001). Se desarrollan en un contexto social donde coexisten diversas concepciones del amor, el sexo, y placer que resulta de la constante interacción física a través de la vinculación erótico- afectiva (Vizzuetth Herrera, García Meraz, \& Guzmán Saldaña, 2013).

Un ingrediente esencial en la vida de la pareja y que se ha considerado un universal cultural (Sánchez Aragón, 2018) es la pasión romántica, misma que desde el momento que surge, provee a los miembros de la diada de una experiencia única que implica un desbordamiento de emociones basadas en el deseo sexual intenso por consumarlo en la entrega sin medida e incluye: enamoramiento, la necesidad de estar cerca del otro constantemente, irracionalidad, amor, el ímpetu, la intrusión obsesiva, la búsqueda desesperada por tener cercanía física continua, la alegría, el erotismo y la sensualidad y reacciones como sentir "mariposas en el estómago". En este escenario, la conexión entre la pasión y el sexo genera un intenso anhelo de unión con el otro que se refleja en la conexión sensual-sexual entre ellos (Sánchez Aragón, 2016).

Trabajos teóricos que han vinculado la personalidad con la sexualidad, han sentado el precedente para ampliar la descripción de dimensiones sexuales. Suponiendo una clave en la identificación de la variación en la sexualidad; en este sentido a partir de los Sexy Seven, otras investigaciones (Díaz Loving, Rivera Aragón, Rocha Sánchez, \& Sánchez Aragón, 2002; Sánchez Aragón, Cruz Martínez, \& Retana Franco, 2006) han sugerido que para garantizar el éxito de la relación, las personas buscan características que perfeccionen o complementen las faltantes.

Tales atributos pueden variar de acuerdo al sexo, es decir, algunas investigaciones (Regan, Levin, Sprecher, Scott, \& Gate, 2000) mencionan que mientras los hombres enfatizan los atributos relacionados a la conveniencia sexual (atractivo, impulso sexual, etc.), las mujeres valoran más características relacionadas con el estatus social (inteligencia, honestidad, etcétera). De manera similar, el sexo femenino se percibe más involucrado emocionalmente y el masculino más erótico, promiscuo, conquistador y perverso (Sánchez Aragón et al., 2006).

Como puede observarse, existen determinadas características que se valoran y resultan atractivas en uno u otro sexo. En este sentido tanto expresiones corporales como faciales,-típicas del coqueteo- generan una interacción compleja (Davis, 2002) que obedece fines preludicos al encuentro sexual y que están encaminados a la obtención de placer y el conocimiento de la pareja.

En este sentido, para Eibl Eibesfeldt (1970) el esquema universal de coqueteo entre hombres y mujeres consiste en mirar fijamente a una posible pareja por algunos segundos, lo que guía el acercamiento entre ambos y favorece la conversación y el contacto. Siendo la combinación del interés sexual y los comportamientos infantiles (como la mirada tímida) indispensables para lograr el encuentro entre ambos, ya que se debe ser capaz de atraer, pero también de dar confianza al otro, siendo las mujeres quienes tienden a expresar este comportamiento.

Algunas investigaciones (Brak Lamy, 2014) muestran que el sexo femenino despierta el deseo a través de gestos, miradas, formas de hablar y uso de lencería mandando señales para ser seducidas. Esta gama de conductas denominadas "seducción”, ponen en juego facetas de personalidad en hombres y mujeres que en otros ámbitos no están presentes, pero que juegan un papel muy importante en el proceso de atracción entre los miembros, donde uno va a propiciar la interacción y el acercamiento con el otro esperando su reciprocidad (Carrasco Chávez, 2010).

Así, las estrategias de seducción constituyen un conjunto de expresiones y manifestaciones que una persona combina con aspectos psicofísicos, comportamentales y de su particular forma de ser (Brak Lamy, 2014) para lograr atraer y encantar a la otra persona con abundancia de estrategias, aperturas y cierres, acercamientos y distanciamientos a partir de las cuales se logra el éxito o fracaso. Esto, precisamente constituye a la comunicación emocional, ya que las conductas generadas (a nivel verbal y no verbal) por un miembro de la pareja producen emociones (positivas o negativas) brindando con ello experiencias, que van desde la intimidad hasta la hostilidad (Sánchez Aragón, 2012; Santos Iglesias et al., 2009) y que hacen sentir al otro individuo erotizado, excitado o apasionado, comunicando y responsabilizándolo de las propias necesidades.

Ejemplos de lo anterior son: las prácticas sexuales (mayor variedad, frecuencia y orgasmo), aspectos socioemocionales (deseo sexual, satisfacción global de la pareja, bienestar marital), conocimientos, actitudes y valores hacia la sexualidad (valores religiosos, actitudes sexuales conservadoras), salud física, vitalidad (enfermedades coronarias, depresión) y barreras ambientales (falta de intimidad) (Frederick, Lever, Gillespie, \& García, 2016; MacNeil \& Byer, 2009; McNulty, Wenner, \& Fisher, 2016).

Por lo tanto, cuando se habla de satisfacción se evalúa la actividad sexual en términos del grado de bienestar y plenitud alcanzado a partir de la respuesta sexual, el deseo, excitación y orgasmo (Carrobles, Gámez Guadix, \& Almendros, 2011). También entendida como la percepción y evaluación que una persona hace de la frecuencia y calidad de su vida sexual, dentro de un continuo que va de lo favorable a lo desfavorable, con base en aspectos individuales, interpersonales y situacionales.

En el terreno de la investigación, diversos estudios como los realizados por Iglesias Campos, Morrell Mengual, Caballero Gascón, Ceccato y Gil Llario (2018), muestran que las mujeres que tienen mayor variedad de prácticas afectivas y sexuales reportan también mayores niveles de satisfacción; por su parte, Johnson, Galambos y Anderson (2015) demuestran que compartir las tareas domésticas y percibir equidad favorece el entendimiento y satisfacción sexual en la pareja; mientras que McNulty et al. (2016) y Schoenfeld, Loving, Pope, Huston y Štulhofer (2017) obtienen correlaciones entre la frecuencia de las relaciones sexuales y la satisfacción de los hombres. 
Así la satisfacción sexual se presenta como un componente central y multideterminado de la relación de pareja (Ahumada, Lüttges, Molina \& Torres, 2014; Fuertes, 2014) y cuya variación a través del tiempo y las situaciones vividas confluyen para impactar de manera tan importante al individuo y sus relaciones de pareja.

Por ello, los objetivos de esta investigación fueron: 1) explorar posibles diferencias o similitudes en las variables de personalidad sexual de la pareja, comunicación emocional de la pasión, estrategias de seducción y satisfacción sexual entre hombres y mujeres, y 2) examinar los efectos de la personalidad sexual de la pareja, comunicación emocional de la pasión, estrategias de seducción sobre la satisfacción sexual en hombres y mujeres por separado.

\section{Método}

\section{Participantes}

A través de una muestra no probabilística propositiva, se reunió un total de 470 participantes heterosexuales pertenecientes a la Ciudad de México (240 mujeres y 230 hombres) con escolaridades de licenciatura (72\%) y bachillerato $(28 \%)$. Su rango de edad oscilaba entre los 15 y 40 años $(M=21.75, D E=3.9)$ y entre 1 y 40 parejas en su historia romántica. Todos reportaron haber experimentado pasión en un periodo de 1 día a 36 meses $(\mathrm{M}=2.28$ meses, $\mathrm{DE}=5.36)$ y éste fue experimentado con la pareja estable $(42.2 \%)$, con un/a amigo/a (25.2\%), con novio/a (15.6\%), con alguien con quien no se tenía ninguna relación (4.9\%), con una expareja (4\%), en una relación abierta $(4 \%)$ y el resto no aclaró ${ }^{1}$.

\section{Instrumentos}

Inventario de Personalidad Sexual de la Pareja (Sánchez Aragón et al., 2006; Cruz Vargas, 2018). Consta de 59 adjetivos con formato de respuesta tipo Likert de cinco opciones, que indican el grado de acuerdo o desacuerdo respecto a qué tanto los participantes creen que su objeto de pasión posee determinados rasgos de personalidad. La versión original (Sánchez Aragón et al., 2006) consta de nueve factores: involucrado emocionalmente, conquistador, atractivo sexual, aventurero, orientación sexual, dispuesto eróticamente, restricción sexual, promiscuo y perverso; sin embargo, la validada actualmente por Cruz Vargas (2018) comprende, 52 adjetivos divididos en seis factores: 1) Promiscuo (p.ej., pervertido, infiel), 2) Involucrado emocionalmente (p.ej., dulce, romántico/a), 3) Atractivo sexual (p.ej., excitante, sexy), 4) Disposición erótica (p.ej., lujurioso/a, desinhibido/a), 5) Orientación

${ }^{1}$ De acuerdo a la literatura, la memoria juega un papel muy importante en la recreación de un evento emocional y dependiendo del tiempo que permanezca en la mente y se recuerde, la emoción se mantendrá (Sánchez Aragón, 2011), por consiguiente, los efectos de la memoria, historia, etcétera, siempre están presentes. de género (p.ej., varonil, caballeroso, femenino/a, hembra)² y 6) Restricción sexual (p.ej., frío/a, puro/a) cuyos coeficientes alpha oscilaron entre .76 y .91 y una varianza total explicada de $52.43 \%$.

Escala de Comunicación Emocional de las Relaciones Románticas (Sánchez Aragón, 2012). Tiene 97 ítems con formato de respuesta tipo Likert pictórico de cuatro puntos indicando la intensidad de la emoción experimentada con base en las conductas realizadas por la pareja/ objeto de pasión. Los factores identificados en esta medida fueron ocho, que explicaron el $49.3 \%$ de la varianza y son: amor-felicidad, pasión, celos, miedo, tristeza, enojo, sorpresa positiva, sorpresa negativa. Debido al objetivo de esta investigación, únicamente se utilizará el componente de pasión que consta de 17 reactivos, y tiene una confiabilidad $\alpha=.93$.

Escala de Estrategias de Seducción (Carrasco Chávez, 2010). Es un auto-reporte dirigido a evaluar las formas de seducción que las personas utilizan para atraer a su pareja con fines sensuales y sexuales. Se conforma por 66 reactivos con formato de respuesta tipo Likert de 5 puntos ( 1 = nunca, $5=$ siempre). Conformado por cuatro dimensiones: 1) Expresión sexual, con 15 reactivos como por ejemplo: “acaricio sus genitales", “jugueteo", "lo/a excito”, 2) Búsqueda de cercanía y permanencia con la pareja que tiene 10 reactivos tales como: "llamo su atención”, "le coqueteo", "me acerco", 3) Expresión y desenvolvimiento con 9 reactivos como: "soy yo mismo/a ", "luzco bien”, "me demuestro seguro/a” y 4) Enamoramiento con 11 reactivos como: "lo/a mimo", "lo/a halago", que explican el $58.9 \%$ de la varianza y con confiabilidades de $\alpha=.94, \alpha=.92, \alpha=.88$ y $\alpha=.87$ respectivamente.

Índice de Satisfacción Sexual (Hudson, 1997; Sánchez Aragón, 2008). Constituido por 25 reactivos con formato de respuesta tipo Likert de 5 puntos que evalúan el grado de acuerdo con una serie de evaluaciones respecto a la propia vida sexual. Sus factores son: 1) Gozo sexual con 10 ítems (p.ej., "Nuestra vida sexual es muy excitante", "Mi pareja es un maravilloso compañero sexual"), 2) Aburrimiento sexual que tiene 8 reactivos (p.ej., "Cuando tenemos sexo es demasiado apresurado y urge terminarlo", "Mi pareja no me satisface sexualmente”), 3) Evasión sexual con 4 reactivos (p.ej., "Mi pareja parece evitar todo contacto sexual conmigo", "Mi pareja no quiere tener relaciones sexuales cuando yo quiero") y 4) Presión sexual con 3 ítems (p.ej., "El sexo con mi pareja se ha vuelto una tarea para mí", "Mi pareja habla demasiado de sexo"). Con una varianza total explicada de $54 \%$ y cuyas confiabilidades para muestra mexicana, son $\alpha=$ $.91, \alpha=.77, \alpha=.71$ y $\alpha=.64$ respectivamente.

\section{Procedimiento}

Los cuestionarios fueron aplicados a la totalidad de participantes por cuatro psicólogos que acudieron a

${ }^{2}$ Cabe señalar que todos los participantes independientemente de su sexo, respondieron al total de los reactivos de cada prueba. 
lugares públicos (plazas comerciales, casas, escuelas, oficinas) para que, de manera voluntaria, anónima y confidencial, respondieran a éstos en el lugar en el que se encontraban y en ese momento. Se les comentaba que era una investigación realizada por una institución educativa pública de prestigio en el país y que se trataba sobre aspectos relacionados a la relación de pareja, momento en el que se les preguntaba si cumplían con el criterio de inclusión de haber experimentado pasión romántica en los últimos seis meses. Ya durante la aplicación, se abrió la posibilidad de resolver dudas y la disposición de los resultados de manera personal.

\section{Resultados}

Para dar cumplimiento al primer objetivo que tenía como fin explorar diferencias o similitudes en las variables, se llevaron a cabo algunas pruebas $t$ de Student para muestras independientes. Los resultados muestran que los hombres perciben a su objeto de pasión mayormente como promiscuo (pervertido, infiel) y restrictivo sexual (frío, puro), mientras que las mujeres lo perciben en su mayoría con orientación de género (varonil, caballeroso). En lo que respecta a las estrategias de seducción, la búsqueda de cercanía y permanencia con la pareja ("Llamo su atención”, "Me acerco"); fue la única que obtuvo diferencias significativas, siendo los hombres quienes más la llevan a cabo. En torno a la comunicación emocional, aburrimiento ("Mi pareja no me satisface sexualmente"), evasión ("Mi pareja parece evitar todo contacto sexual conmigo") y presión sexual ("El sexo con mi pareja se ha vuelto una tarea para mí”) son los hombres quienes puntúan más alto (ver Tabla 1).

Para dar cumplimiento al segundo objetivo, se llevaron a cabo análisis de correlación producto-momento de Pearson para mujeres y hombres por separado. Los hallazgos obtenidos muestran que, en la medida en que las mujeres perciben que su objeto de pasión tiene más rasgos de personalidad sexual como: estar dispuesto eróticamente (cachondo, complaciente), atractivo (apantallador y apasionado), involucrado emocionalmente (adorable y amoroso) y con orientación masculina (varonil, caballeroso), tienden a experimentar más gozo sexual ("Nuestra vida sexual es muy excitante", "Mi pareja es un maravilloso compañero sexual”). Asimismo, cuando ellas experimentan pasión por las conductas que realiza dicha persona (comunicación emocional de la pasión) aunado a la ejecución de estrategias de seducción como expresión y desenvolvimiento ("Soy yo misma”, "Luzco bien"), expresión sexual (“Acaricio sus genitales”, “Jugueteo”), búsqueda de cercanía ("Llamo su atención”, "Le coqueteo”) y enamoramiento ("Lo mimo”, "Lo halago"), se evaluará la vida sexual como gozosa.
Tabla 1.

Diferencias sexuales en torno a las variables personalidad sexual, comunicación emocional, estrategias de seducción y satisfacción sexual.

\begin{tabular}{|c|c|c|c|}
\hline & Hombres & Mujeres & $t, p$ \\
\hline Promiscuo & 2.31 & 2.07 & $\mathrm{t}=-2.698, \mathrm{p}=.007$ \\
\hline $\begin{array}{l}\text { Orientación de } \\
\text { género }\end{array}$ & 2.38 & 3.74 & $\mathrm{t}=15.389, \mathrm{p}=.000$ \\
\hline Restricción sexual & 2.65 & 2.11 & $\mathrm{t}=-7.185, \mathrm{p}=.000$ \\
\hline $\begin{array}{l}\text { Comunicación } \\
\text { Emocional }\end{array}$ & 2.69 & 2.52 & $\mathrm{t}=-2.791, \mathrm{p}=.005$ \\
\hline
\end{tabular}

\begin{tabular}{|c|c|c|c|}
\hline $\begin{array}{l}\text { Búsqueda de } \\
\text { cercanía y } \\
\text { permanencia }\end{array}$ & 3.93 & 3.76 & $\mathrm{t}=-2.298, \mathrm{p}=.022$ \\
\hline Aburrimiento & 2.73 & 2.33 & $\mathrm{t}=-5.281, \mathrm{p}=.000$ \\
\hline Presión & 2.88 & 2.37 & $\mathrm{t}=-5.194, \mathrm{p}=.000$ \\
\hline Evasión & 2.41 & 1.83 & $\mathrm{t}=-5.686, \mathrm{p}=.000$ \\
\hline
\end{tabular}

También se observó que, a más uso de estrategias de seducción como la búsqueda de cercanía, expresión sexual y desenvolvimiento, hay una tendencia al aburrimiento sexual ("Cuando tenemos sexo es demasiado apresurado y urge terminarlo", "Mi pareja no me satisface sexualmente”). Mientras que cuando las mujeres perciben en su objeto de pasión más rasgos de personalidad sexual como: promiscuo (pervertido, infiel), dispuesto eróticamente y restringido sexualmente (lujurioso, desinhibido y frío, casto), además de ejecutar la estrategia de seducción de expresión sexual, hay más presión sexual ("El sexo con mi pareja se ha vuelto una tarea para mí", "Mi pareja habla demasiado de sexo"). Por último, en la medida en que las mujeres perciben al objeto de pasión como promiscuo, ellas sienten mayor evasión sexual ("Mi pareja parece evitar todo contacto sexual conmigo", "Mi pareja no quiere tener relaciones sexuales cuando yo quiero") (ver Tabla 2). 
Tabla 2.

Correlación entre Personalidad sexual, Comunicación Emocional y Estrategias de Seducción con Satisfacción Sexual en Mujeres.

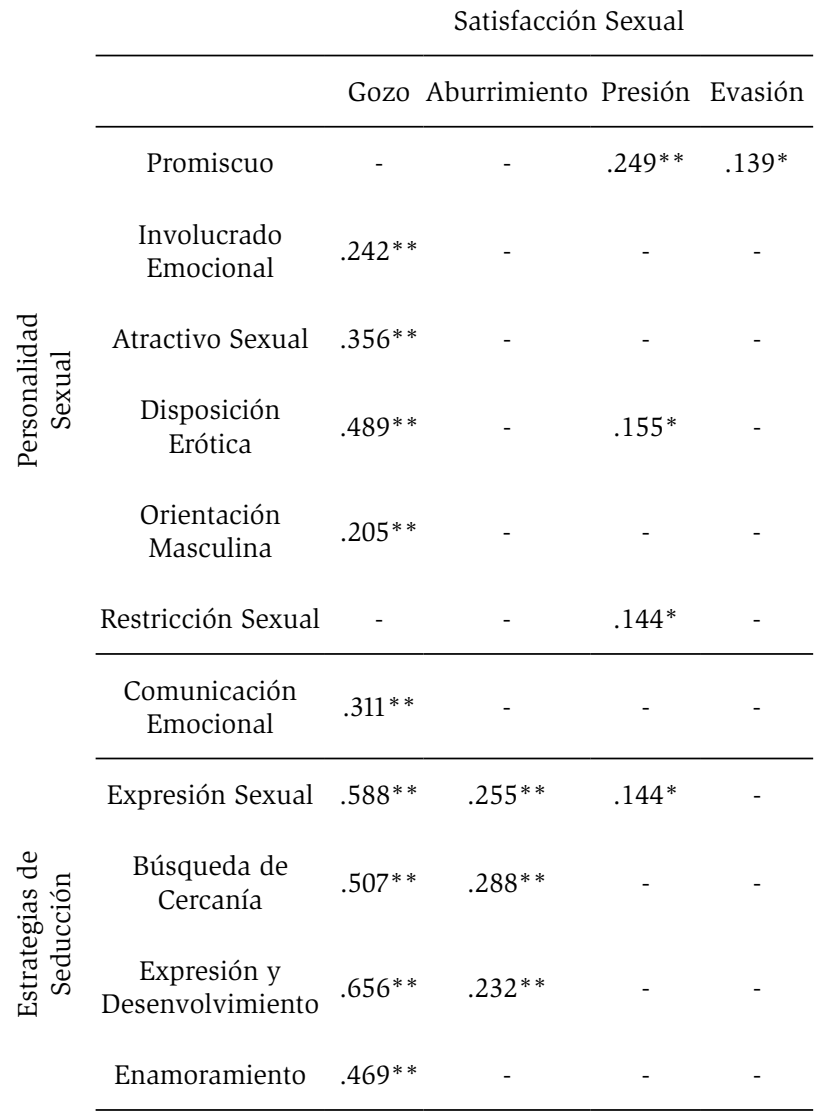

** Correlación significativa a .01 (bilateral)

* Correlación significativa a .05 (bilateral)

Para el caso de los varones, cuando estos perciben a las mujeres con rasgos como atractivo sexual, disposición erótica e involucrada emocionalmente, entonces sienten más gozo; asimismo cuando en la intimidad usan estrategias de seducción como la expresión sexual, desenvolvimiento, búsqueda de cercanía, y enamoramiento.

Por su parte, el aburrimiento sexual tiene lugar en los hombres cuando perciben a su objeto de pasión como más promiscua, restringida sexualmente, con orientación masculina y no involucrada emocionalmente; aunado a experimentar pasión por las conductas que realiza y restringir el uso del enamoramiento y expresión como estrategias de seducción. Asimismo, para que los hombres sientan presión y evasión sexual, la percepción del objeto de pasión es la misma (promiscua, restrictiva sexualmente y con orientación masculina); incorporando en la primera la disposición erótica, y en la segunda, poco involucramiento emocional y atractivo sexual, además de la restricción en el uso del enamoramiento y expresión y desenvolvimiento como estrategias de seducción (ver Tabla 3).
Tabla 3.

Correlación entre Personalidad sexual, Comunicación Emocional y Estrategias de Seducción con Satisfacción Sexual en Hombres.

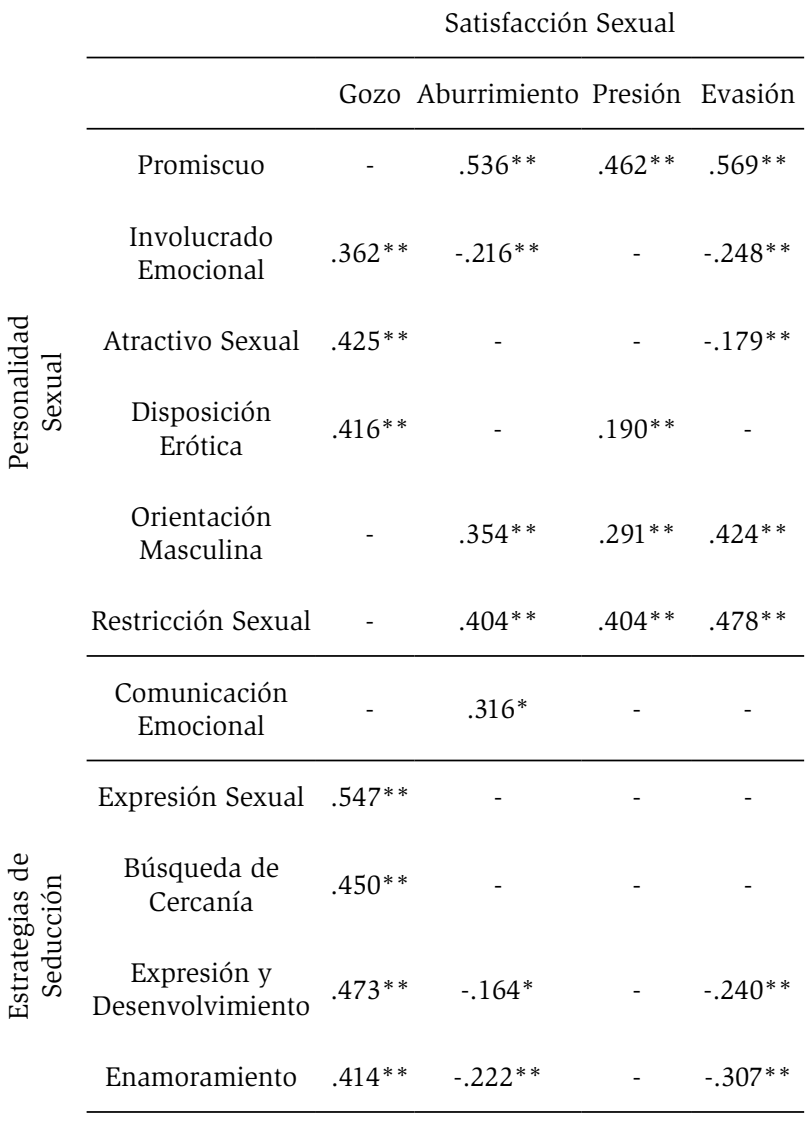

** Correlación significativa a .01 (bilateral)

* Correlación significativa a .05 (bilateral)

\section{Discusión}

La presente investigación se encaminó a examinar el papel del sexo en la manera en la que se experimenta una serie de variables vinculadas a la satisfacción sexual como lo son: la personalidad sexual percibida en el objeto de pasión, la comunicación emocional y las estrategias de seducción. Para ello, se realizaron inicialmente unos análisis $t$ de Student que permitieron identificar las diferencias existentes en dichas variables. Los hallazgos muestran que los hombres perciben características como la promiscuidad y la restricción sexual en su objeto de pasión, es decir, a una persona que a la vez es pervertida o infiel, pura y casta. Dicho resultado resulta congruente con Reid y Bing (2000) quienes mencionan la clásica representación de las mujeres como Madonas sexuales buenas y malas; las primeras descritas como vírgenes, puras y castas, mientras que las segundas son consideradas como promiscuas y seductoras. 
Aunado a lo anterior, Regan y Dreyer (2008), Gray, García y Gesselman (2018) encontraron que las conductas sexuales de hombres se caracterizan por mayor receptividad de ofertas sexuales casuales - relacionados a la experimentación, placer físico y consumo de alcohol - y el inicio temprano de la vida sexual con parejas en promedio cinco años mayor; lo que puede dar pie a percibir características de personalidad más perversas, indecentes, vulgares y polígamas.

En el caso del sexo femenino, ellas perciben a su objeto de pasión como más orientado al género (varonil, caballeroso), lo que se apoya en lo obtenido por Regan et al. (2000), quienes identificaron que, en la selección de una pareja sexual para corto plazo, las mujeres valoraron más las características relacionadas al estatus social, mientras que ellos se enfocaron en atributos relacionados con la deseabilidad sexual.

En lo que respecta a las estrategias de seducción, llamar la atención de la pareja y acercarse a ella (búsqueda de cercanía y permanencia con la pareja) son de las acciones que los hombres más llevan a cabo en comparación con las mujeres. Respecto a esto, Álvarez Yguarán et al. (1990) destacan que las percepciones propias de los hombres se orientan a la "exclusividad" en la iniciativa de conductas eróticas, facilitando al sexo opuesto su “colaboración", propiciando excitación y deseo femenino; mientras que la búsqueda de cercanía física - usando ademanes indirectos o la verbalización- son conductas llevadas a cabo con el fin de generar excitación y deseo en su contraparte femenina.

En la comunicación emocional también se observaron diferencias que indican que son también los hombres quienes más pasión experimentan ante los comportamientos que leen en su objeto de pasión. Esto resulta coherente con hallazgos (Álvarez Yguarán et al.,1990; Eichenlaub, 1961; Ellis, 1963) que mencionan que el placer experimentado por los hombres se debe a que son conscientes del gozo que producen en su objeto de pasión y la importancia que le confieren a la verbalización y ademanes -como miradas excitantes, palabras sugerentes, contacto físico o algún sonido- durante la estimulación erótica que, a su vez, funciona como indicador de autenticidad en la respuesta (López Sánchez, 2004).

En lo que respecta a la Satisfacción Sexual, las diferencias tienen que ver con que el sexo masculino considera que su pareja no lo satisface sexualmente (aburrimiento), evita todo contacto con él (evitación) y que el sexo se ha vuelto una tarea (presión), lo cual se vincularía, en parte, con el hecho de que perciban a su objeto de pasión como restrictiva sexualmente (observado al inicio de los resultados). Lo anterior resulta lógico, dada la erótica masculina que se caracteriza por centrarse de manera exclusiva en la "genitalidad" (Álvarez Gayou, 1996) llegando a la intimidad con demasiada intensidad o rapidez. Esto hace que, por una parte, la evaluación de la satisfacción no vaya más allá del evento sexual y, por otra, favorece la percepción de frialdad, generando inhibición en la expresión sexual y rechazo en su contraparte femenina.

En cuanto a los resultados obtenidos para cumplir con el segundo objetivo, por medio de los análisis de correlación, las mujeres mostraron que, en la medida en que perciben que su objeto de pasión tiene más rasgos de personalidad sexual como: dispuesto eróticamente, atractivo, involucrado emocionalmente y con orientación masculina, tienden a experimentar más gozo sexual. Congruente con investigaciones (Regan et al., 2000) que demuestran relaciones entre la satisfacción sexual y la presencia de ciertas características en el otro.

Asimismo, cuando ellas experimentan pasión por las conductas que realiza dicha persona aunado a la ejecución de estrategias de seducción como expresión y desenvolvimiento, expresión sexual, búsqueda de cercanía y enamoramiento, se evaluará la vida sexual como gozosa. Esto podría deberse a que cuando la participante siente pasión por las conductas del otro, - que incluyen aspectos subjetivos, no estrictamente sexuales- pasa de un estado neutro a uno de excitación (Basson, 2002); en el que el deseo y necesidad intensa por acercarse al otro permiten el despliegue de estrategias necesarias para seducir.

Además, el conocimiento y estimulación sensual de su cuerpo, la experiencia de libertad en la relación y toma de iniciativa en el contacto erótico, facilita el disfrute sexual para algunas mujeres. Esta visión general es postulada por Ortiz Barón, Gómez Zapiain y Apodaca (2002) y más recientemente para Stulhofer, Ferreira y Landripet (2014), para quienes la vinculación afectiva, la similitud o complementariedad y las expectativas cumplidas por la pareja son algunos de los factores más importantes para alcanzar la satisfacción sexual.

También se observó que, a mayor uso de estrategias de seducción (como la búsqueda de cercanía, expresión sexual y desenvolvimiento), hay una tendencia al aburrimiento sexual. El hecho de que todo dependa de un solo miembro resulta tedioso, lo cual resulta congruente con lo propuesto por Brack Lamy (2015), quien define la seducción como una gama de conductas donde uno propicia la interacción y el acercamiento con el otro, esperando su correspondencia. Similar a lo señalado por Alberoni (1986), quien reporta que las mujeres que no se sienten deseadas, amadas o con un esfuerzo renovado de seducción sufren decepción.

Mientras que cuando las mujeres perciben en su objeto de pasión más rasgos de personalidad sexual como: ser promiscuo, dispuesto eróticamente y restringido sexualmente, además de ejecutar la estrategia de seducción de expresión sexual, hay más presión sexual ("El sexo con mi pareja se ha vuelto una tarea para mí”, "Mi pareja habla demasiado de sexo"). Al respecto, Valdez Medina et al., (2007) mencionan que, al no tener la certeza de que la pareja sea la indicada para relacionarse afectivamente, dadas sus características y comportamientos, se generará duda respecto al deseo que siente el otro, representando un inhibidor de la conducta sexual

A la vez, en la medida en que las mujeres perciben al objeto de pasión como promiscuo, ellas sienten mayor evasión sexual ("Mi pareja parece evitar todo contacto sexual conmigo", "Mi pareja no quiere tener relaciones sexuales cuando yo quiero"). En torno a esto, hay que 
señalar el hecho de que las mujeres unen el amor con el sexo, lo que Sprecher (2002) apoya, señalando que ellas necesitan en mayor medida sentirse amadas para poder tener relaciones sexuales, aunado a factores como el compromiso y la estabilidad que brindan la certeza y seguridad en el vínculo y que resultan indispensables para alcanzar la satisfacción sexual. El hecho de que se perciba al otro como promiscuo, definitivamente repele y aleja a las participantes.

Para el caso de los varones, cuando éstos perciben a las mujeres con rasgos como atractivo sexual, disposición erótica e involucrada emocionalmente, sienten más gozo. Esto porque dichas características garantizan la reciprocidad y correspondencia en el deseo y sentir durante la interacción sexual. Además, en este contexto, el despliegue de estrategias de seducción (expresión sexual, desenvolvimiento, búsqueda de cercanía, y enamoramiento) incrementan la intimidad (Alberoni, 1986) y, por tanto, el gozo sexual. Hay que reparar en el punto de que justamente el gozo sexual puede resultar mayor, debido a que los hombres tienen la capacidad de desligar el sexo como acto placentero del amor y que sus conductas sexuales se ven motivadas por su pareja a través de sus sentidos, la sensación de afecto, gusto por su arreglo, e incitar la excitación física (Valdez Medina et al., 2007).

Mientras que el aburrimiento, la presión y la evasión sexual tienen lugar en la medida en que los hombres perciben a sus objetos de pasión como promiscua, restringida sexualmente, con orientación masculina, no involucrada emocionalmente, así como la experimentación ante sus conductas y menor uso de estrategias de enamoramiento y desenvolvimiento. Esto es congruente con la idea de que un estímulo de esa naturaleza (percepción de promiscuidad, restricción, orientación masculina y no involucramiento emocional) no favorece el deseo ni una visión positiva en la experimentación de gozo, siendo la desvinculación afectiva una de sus principales causas (Heiman, Long, Smith, Fisher, \& Sand, 2011). La falta de atributos, sumado a la carencia de habilidades de seducción, produce un fracaso ante lo esperado, pues la interacción que se da tiene un tono de desinterés y tedio (Ortiz Ortega, Rivas Zivy, Huerta, Salazar, \& Gómez González, 2004).

Recapitulando lo anterior, se concluye que para que hombres y mujeres sientan gozo sexual, los ingredientes esenciales son: el percibir al objeto de pasión con características como: involucrado/a emocional, atractivo/a sexual y dispuesto/a eróticamente, así como la ejecución de estrategias como expresión sexual, búsqueda de cercanía, expresión y desenvolvimiento y enamoramiento. Mientras que percibir a éste como promiscuo o restringido sexualmente y el bajo o constante uso de las mismas estrategias de seducción, favorece los matices negativos de la satisfacción sexual como la evasión, la presión y el aburrimiento. Esto resulta relevante al recordar que la dinámica de la pareja tanto en la intimidad como en otras esferas requiere de la novedad (Sánchez Aragón, 2016), renovación y creatividad para resultar interesantes a sus miembros y asegurar la experiencia emocional y física lo más satisfactoria posible. Esta investigación supone un aporte al conocimiento teórico del tema, así como a la solución de problemas prácticos que en la vida cotidiana de las parejas resulta fundamental.

Al respecto, hay que recordar que las diferencias entre sexos tienen un impacto no solo en la experimentación de la satisfacción sexual, también en la forma de relacionarse sexualmente (Valdés Rodríguez, Sapién López, \& Córdoba Basulto, 2004). Los resultados descritos integran variables tanto individuales como relacionales que resuelven la necesidad de explorar el clima interpersonal no sexual (Schoenfeld et al., 2017) permitiendo comprender la complejidad del constructo estudiado, constatando las diferencias sexuales particulares en una muestra mexicana.

Entre las limitaciones de la investigación, es preciso mencionar que, si bien la metodología empleada permitió alcanzar los objetivos principales, algunas de las características de la muestra, específicamente la persona por la que experimentaron pasión (amigo/a, novio/a, esposo/a y otros), pudo haber generado ligeras diferencias no exploradas por tratarse de grupos no homogéneos. Partiendo de lo anterior, algunas de las líneas futuras, consisten en explorar posibles diferencias entre tales grupos, para intentar identificar si determinadas características funcionan como predictores destacados en la satisfacción sexual de los participantes. Aunado a lo anterior, se encuentra reunir muestras con otras orientaciones sexuales para explorar posibles diferencias.

\section{REFERENCIAS}

Ahumada, S., Lüttges, C., Molina T., \& Torres, S. (2014). Satisfacción sexual: revisión de los factores individuales y de pareja relacionados. Revista Hospital Clínico, 1(25), 278-284.

Alberoni, F. (1986). El erotismo. Barcelona, ES: Gedisa.

Álvarez Gayou, J. L. (1996). El acto sexual. En M. E. Gómez López (Ed.), Sexualidad Humana de McCary (pp. 125-137). México D.F., MX: Manual Moderno.

Álvarez Yguarán, F., Gómez, L. E., Acosta, C., Eijaiek de Manzano, L., Saade de Vigna, M., \& Vargas Rozo, P. (1990). Estudio descriptivo del comportamiento sexual de hombres y mujeres que mantienen relación de pareja en Barranquilla. Investigación y Desarrollo, 1(1), 31-35.

Basson, R. (2002). A model of women's sexual arousal. Journal of Sex \& Marital therapy, 28(1), 1-10. doi:10.1080/0092623023172 50963

Brak Lamy, G. (2014). Heterosexual Seduction in the Urban Night Context: Behaviors and Meanings. The Journal of Sex Research, 52(6), 690-699. doi:10.1080/00224499.2013.856835

Campos, I., Morell Mengual, P., Caballero Gascón, V., Ceccato, L., \& Gil Llario, M. D. (2018). Satisfacción sexual femenina: Influencia de la edad y variedad de prácticas sexuales. International Journal of Development and Educational Psychology, $1(1), 3-7$.

Carrobles, J., Gámez-Guadix, M. \& Almendros, C. (2011). Funcionamiento sexual, satisfacción sexual y bienestar psicológico y subjetivo en una muestra de mujeres españolas. Anales de Psicología, 27(1), 27-34. 
Carrasco Chávez, E. (2010). Seducción: una forma de expresión emocional femenina (Tesis de Licenciatura en Psicología inédita). Universidad Nacional Autónoma de México, México.

Cruz Vargas, D. J. (2018). Satisfacción Sexual: Identificando el efecto de la Personalidad, Comunicación emocional, Estrategias de regulación, y Cercanía con el objeto de pasión (Tesis de Licenciatura en Psicología inédita). Universidad Nacional Autónoma de México, México.

Díaz Loving, R., Rivera Aragón, S., Rocha Sánchez, T. E. \& Sánchez Aragón, R. (2002). Marcado por la conquista: Rasgos de Personalidad derivados de la vida sexual. Revista de Psicología Social y Personalidad, 18(1), 77-92.

Eibl Eibesfeldt, I. (1970). Ethology: The biology of behavior. New York, NY: Richart and Winston.

Eichenlaub, J. E. (1961). The marriage art. New York, NY: Dell.

Ellis, A. (1963). Sex and the single man. New York, NY: Lyle Stuart.

Frederick, D., A., Lever, J., Gillespie, J. B., \& García, J. R. (2016). What keeps passion alive? Sexual Satisfaction is associated with sexual communication, mood setting, sexual variety, oral sex, orgasm, and sex frequency in a national U.S. Study. The Journal of Sex Research, 54(2), 1-16. doi:10.1080/00224499 .2015 .1137854

Fuertes, A. (2014). Affective relationships and sexual satisfaction in couples. International Journal of Social Psychology, 15(3) 343-356. doi:10.1174/021347400760259758

Gray, P. B., García, J. R., \& Gesselman, A. N. (2018). Age-related patterns in sexual behaviors and attitudes among single U.S. Adults: An evolutionary approach. Evolutionary Behavioral Sciences. Advance online publication. doi:10.1037/ebs0000126

Heiman, J., Long, S. J., Smith, S.N., Fisher, W. A., \& Sand, M. S. (2011). Sexual Satisfaction and relationship happiness in midlife and older couples in five countries. Archives of Sexual Behavior, 40(4),711-753. doi:10.1007/s10508-010-9703-3.

Iglesias Campos, P., Morell Mengual, V., Caballero Gascón, V., Ceccato, R. \& Gil Llario, M. D. (2018). Satisfacción sexual femenina: influencias de la edad y varied de prácticas sexuales. International Journal of Developmental and Educational Psychology, 1(1), 85-92. doi:10.17060/ijodaep.2018.n1.v1.1163

Johnson, M. D., Galambos, N. L., \& Anderson, J. (2015). Skip the dishes? Not so fast! Sex and housework revisited. Journal of Family Psychology, 30(2), 203-213. doi:10.1037/fam0000161

López Sánchez, F. (2004). Conducta Sexual de Mujeres y Varones: Iguales y Diferentes. En E. Barberá, I. M., Benlloch (Eds.), Psicología y Género (pp. 145-192). Madrid, ES: Pearson, Prentice Hall.

MacNeil, S. \& Byers, E. S. (2009). Role of sexual self-disclosure in the sexual satisfaction of long-term heterosexual couples. The Journal of Sexual Research, 46(1), 3-14.

McNulty, J. K., Wenner, C. A., \& Fisher, T., D. (2016). Longitudinal Association among relationships satisfaction, sexual satisfaction, and frequency of sex in early marriage. Archives of Sexual Behavior, 45(1), 85-97. doi:10.1007/s10508-014-0444-6

Ortiz Barón, M. J., Gómez Zapiain, J. \& Apodaca, P. (2002). Apego y satisfacción afectivo-sexual. Psicothema, 14(2), 469-475.

Ortiz Ortega, A., Rivas Zivy, M., Huerta, F., Salazar G., \& Gómez González, A. (2004). El papel de los hombres en el ejercicio y apropiación de los derechos sexuales y reproductivos de las mujeres. Perinatología y Reproducción humana, 18(1), 23-33.
Regan, P. C. \& Dreyer, C.S. (2008). Lust? Love? Status? Young adult's motives for engaging in casual sex. Journal of Psychology \& Human Sexuality, 11 (1),1-24. doi: 10.1300/J056v11n01_01

Regan, P. C., Levin, L., Sprecher, S., Scott, C., \& Gate, R. (2000). Partner preferences. What characteristics do men and woman desire in their short-term sexual and long-term romantic partners? Journal of Psychology \& Human Sexuality, 12(3), 1-21. doi: 10.1300/J056v12n03_01

Reid, P. T. \& Bing, V. M. (2000). Sexual roles of girls and women: An ethnocultural lifespan perspective. In C. B. Travis \& J.W. White (Eds.), Sexuality, society, and feminism (pp. 141-166). Washington, DC: American Psychological Association. doi: 10.1037/10345-006

Ruiz Becerril, D. (2001). Relaciones de Pareja. Revista de Educación, 325, 49-57.

Sánchez Aragón, R. (2012). Comunicación Emocional en las Relaciones Románticas: Validación de una Medida en México. Interpersona, 6(2), 211-226.

Sánchez Aragón, R. (2016). La Pareja Romántica en México. Desentrañando su naturaleza comunicativa y emocional. México: Universidad Nacional Autónoma de México.

Sánchez Aragón, R. (2018). Caracterización psicológica de la pasión romántica. Pensando Psicología, 14(24) [en prensa].

Sánchez Aragón, R., Cruz Martínez, L. \& Retana Franco, B. (2006). ¿Cómo soy en el Ámbito Sexual y cómo percibo a mi objeto de pasión? En R. Sánchez Aragón, R. Díaz Loving y S. Rivera Aragón (Eds.), La Psicología Social en México (pp. 860868). Tabasco, MX: Universidad Juárez Autónoma de Tabasco.

Santos Iglesias, P., Sierra, C., García, M., Martínez, A., Sánchez, A., \& Tapia, I. (2009). Índice de Satisfacción Sexual (ISS): un estudio sobre su fiabilidad y validez. International Journal of Psychology and Psychological Therapy, 9(2), 259-273.

Schoenfeld, E. A., Loving, T. J., Pope, M. T., Huston, T. L., \& Štulhofer, A. (2017). Does sex really matter? Examining the Connections between spouse's nonsexual behaviors, sexual frequency, sexual satisfaction, and marital satisfaction. Archives Sexual Behavior, 46(2), 489-501. doi:10.1007/s10508015-0672-4.

Sprecher, S. (2002). Sexual Satisfaction in premarital relationships: Associations with satisfaction, love, commitment and stability. The journal of sex research, 39(3), 190-196. doi:10. 1080/00224490209552141

Stulhofer, A., Ferreira, L. C., \& Landripet, I. (2014). Emotional intimacy sexual desire, and sexual satisfaction among partnered heterosexual men. Sexual and Relationships Therapy, 29(2), 229-244. doi:10.1080/14681994.2013.870335

Valdez Medina, J. L., Arce Valdez, J., Álvarez Valdez, J. E., González Sánchez, M., Corza Fierro, F. J., \& González Arratia, N. I. (2007). Sexología, motivación y desmotivación sexual en parejas con unión conyugal. Un análisis por sexo. Archivos Hispanoamericanos de Sexología, 13(2), 151-162.

Valdés Rodríguez, M., Sapién López, J., \& Córdoba Basulto, D. (2004). Significados de satisfacción sexual en hombres y mujeres de la zona metropolitana. Psicología y Ciencia Social, 6(1), 34-48.

Vizzuetth Herrera, A., García Meraz, M., \& Guzmán Saldaña, R. (2013). Significado psicológico de los constructos novios, amigovios y free para jóvenes pachuqueños. PSICUMEX, 3(2), 4-14. 


\section{Inventario de Personalidad Sexual de la Pareja}

INSTRUCCIONES. Por favor, evalúe que tan exacto es cada uno de los siguientes adjetivos para describir a

Describa cómo es actualmente y en general, no como te gustaría que fuera. Después de cada adjetivo, por favor anote un número indicando que tan exacta o inexacta es esa palabra para describir a esa persona, utilizando la siguiente escala.

\begin{tabular}{|c|c|c|c|c|c|}
\hline & & \multicolumn{3}{|c|}{ Muy Exacto } & 5 \\
\hline & \multicolumn{3}{|c|}{ Algo Exacto } & 4 & \\
\hline & \multicolumn{2}{|c|}{ Ni exacto ni Inexacto } & 3 & & \\
\hline \multicolumn{2}{|c|}{ Algo Inexacto } & \multirow[t]{2}{*}{2} & & & \\
\hline Muy Inexacto & 1 & & & & \\
\hline 1. Indecente & 1 & 2 & 3 & 4 & 5 \\
\hline 2. Infiel & 1 & 2 & 3 & 4 & 5 \\
\hline 3. Involucrado(a) emocionalmente & 1 & 2 & 3 & 4 & 5 \\
\hline 4. Lujurioso(a) & 1 & 2 & 3 & 4 & 5 \\
\hline 5. Macho & 1 & 2 & 3 & 4 & 5 \\
\hline 6. Masculino(a) & 1 & 2 & 3 & 4 & 5 \\
\hline 7. Masoquista & 1 & 2 & 3 & 4 & 5 \\
\hline 8. Moralista & 1 & 2 & 3 & 4 & 5 \\
\hline 9. Mujeriego(a) & 1 & 2 & 3 & 4 & 5 \\
\hline 10. Obsceno(a) & 1 & 2 & 3 & 4 & 5 \\
\hline 11. Perverso(a) & 1 & 2 & 3 & 4 & 5 \\
\hline 12. Pervertido(a) & 1 & 2 & 3 & 4 & 5 \\
\hline 13. Polígamo(a) & 1 & 2 & 3 & 4 & 5 \\
\hline 14. Promiscuo(a) & 1 & 2 & 3 & 4 & 5 \\
\hline 15. Protector(a) & 1 & 2 & 3 & 4 & 5 \\
\hline 16. Puro(a) & 1 & 2 & 3 & 4 & 5 \\
\hline 17. Religioso(a) & 1 & 2 & 3 & 4 & 5 \\
\hline 18. Reprimido(a) sexualmente & 1 & 2 & 3 & 4 & 5 \\
\hline 19. Romántico(a) & 1 & 2 & 3 & 4 & 5 \\
\hline 20. Sádico(a) & 1 & 2 & 3 & 4 & 5 \\
\hline 21. Sensual(a) & 1 & 2 & 3 & 4 & 5 \\
\hline 22. Sexy & 1 & 2 & 3 & 4 & 5 \\
\hline 23. Tierno(a) & 1 & 2 & 3 & 4 & 5 \\
\hline 24. Varonil & 1 & 2 & 3 & 4 & 5 \\
\hline
\end{tabular}

\begin{tabular}{|c|c|c|c|c|c|}
\hline 25. Virginal & 1 & 2 & 3 & 4 & 5 \\
\hline 26. Vulgar & 1 & 2 & 3 & 4 & 5 \\
\hline 27. Adorable & 1 & 2 & 3 & 4 & 5 \\
\hline 28. Adultero(a) & 1 & 2 & 3 & 4 & 5 \\
\hline 29. Afectuoso(a) & 1 & 2 & 3 & 4 & 5 \\
\hline 30. Amoroso(a) & 1 & 2 & 3 & 4 & 5 \\
\hline 31. Apasionado(a) & 1 & 2 & 3 & 4 & 5 \\
\hline 32. Atractivo(a) & 1 & 2 & 3 & 4 & 5 \\
\hline 33. Atractivo(a) sexualmente & 1 & 2 & 3 & 4 & 5 \\
\hline 34. Atrayente & 1 & 2 & 3 & 4 & 5 \\
\hline 35. Atrevido(a) & 1 & 2 & 3 & 4 & 5 \\
\hline 36. Caballeroso(a) & 1 & 2 & 3 & 4 & 5 \\
\hline 37. Cachondo(a) & 1 & 2 & 3 & 4 & 5 \\
\hline 38. Cálido(a) & 1 & 2 & 3 & 4 & 5 \\
\hline 39. Cariñoso(a) & 1 & 2 & 3 & 4 & 5 \\
\hline 40. Casto(a) & 1 & 2 & 3 & 4 & 5 \\
\hline 41. Cínico (a) & 1 & 2 & 3 & 4 & 5 \\
\hline 42. Sinvergüenza(a) & 1 & 2 & 3 & 4 & 5 \\
\hline 43. Conquistador(a) & 1 & 2 & 3 & 4 & 5 \\
\hline 44. Desinhibido(a) & 1 & 2 & 3 & 4 & 5 \\
\hline 45. Dispuesto(a) eróticamente & 1 & 2 & 3 & 4 & 5 \\
\hline 46. Dulce & 1 & 2 & 3 & 4 & 5 \\
\hline 47. Erótico(a) & 1 & 2 & 3 & 4 & 5 \\
\hline 48. Excitante & 1 & 2 & 3 & 4 & 5 \\
\hline 49. Femenino(a) & 1 & 2 & 3 & 4 & 5 \\
\hline 50. Frío(a) & 1 & 2 & 3 & 4 & 5 \\
\hline 51. Gusto por las orgías & 1 & 2 & 3 & 4 & 5 \\
\hline 52. Hembra & 1 & 2 & 3 & 4 & 5 \\
\hline
\end{tabular}

